\title{
Integration of local features as a function of global goodness and spacing
}

\author{
MARIA I. LASAGA and HEIKO HECHT \\ University of Virginia, Charlottesuille, Virginia
}

\begin{abstract}
In two experiments, the accuracy with which subjects detected a conjunction of features was examined as a function of the spacing between items and the goodness of the axis along which they were located. In each array, two items were arranged along a vertical, a horizontal, or a diagonal axis. Based on the well-established oblique effect, the vertical and horizontal axes were considered to be good global patterns and the diagonals were considered to be poor. In Experiment 1 , the two items in an array could be two horizontal lines, two vertical lines, a vertical and a horizontal line, or a plus sign with one of the single lines. In Experiment 2, a positiveand a negative-diagonal line were used as the individual features, and an " $X$ " was used as the conjunction. The results from Experiment 1 indicated that global goodness influenced only the rate of illusory conjunctions, and not of feature errors. Ilusory conjunctions of vertical and horizontal line segments were more likely to occur in vertical and horizontal arrangements. The results from Experiment 2 revealed a reversal of the effect of global goodness on the rate of illusory conjunctions: Illusory conjunctions of negative- and positive-diagonal line segments were more likely to occur in diagonal arrangements. The results of both experiments taken together showed the existence of an important and new factor that influences the likelihood that features of shape will be conjoined: the ease with which line segments conjoin when they are translated along their extent toward each other. In both experiments, greater spacing between items produced more feature-identification errors and fewer feature-integration errors than did less spacing.
\end{abstract}

Many theories of pattern perception have been concerned with the contribution of lower level, or local, information to the processing of higher level, or global, information, and vice versa (e.g., Neisser, 1967; Treisman \& Gelade, 1980; Uhr, 1966). Empirical examinations of this issue have typically addressed the question of whether global information or local information is available earlier, by measuring how quickly or accurately local versus global information can be identified in the same stimulus (e.g., Kinchla \& Wolfe, 1979; Martin, 1979; Navon, 1977). However, these studies have been unable to determine whether more quickly processed information is simply available earlier but independently in the perceptual system, or whether it is actually used as input, in full or in part, in order to process information at the level completed later (Lasaga, 1989; Navon, 1981; Ward, 1983).

Pomerantz $(1981,1983)$ has pointed out that at least some information about local units is logically required as input in order to identify any global pattern. This point applies even when the local units are physically disconnected from each other and their identity is not critical for the perceived global contour (e.g., a letter " $T$ " formed out of pebbles); we will refer to such a stimulus as a

The authors wish to thank $J$. Letizio and $C$. Hattangadi for their assistance in running subjects in this study. We also wish to thank Jack Gallant for his helpful critical comments on an earlier version of this manuscript. Reprint requests should be sent to Maria I. Lasaga, Department of Psychology, Gilmer Hall, University of Virginia, Charlottesville, VA 22903 grouping stimulus. Even in such a case, the registration of some stimulus energy at each location where a local unit occurs is still logically required to identify the global pattern.

In contrast, identification of local units in a grouping stimulus can logically be accomplished without any global input whatsoever. Nonetheless, observers often identify the global pattern more quickly than they identify the local units of these stimuli (e.g., Kinchla \& Wolfe, 1979; Navon, 1977). It would seem efficient for the visual system to take advantage of earlier global information during later local processing, even if it is not logically (i.e., structurally) required to do so. Yet the psychological reality of such a contribution has not been established.

In this paper, we will examine the specific possibility that global information can help to specify where the local units of a grouping stimulus are located for further local processing. This proposed type of global input is simply the flip side of the relationship just described between global identity and the location of local units: If information about the global contour requires information about where the local units are located, then, when completed earlier, the global level could provide this information about location. Such location information, if any is provided, must itself be global (i.e., relative) in nature. Otherwise, providing information to the local level that is already available at that level would not constitute a processing advantage.

That human perceivers can process the location of local units of grouping stimuli in a relative fashion has been 
demonstrated by research on pattern goodness. Several of these studies (e.g., Attneave, 1955; Bear, 1973; Schnore \& Partington, 1967) have shown that the location of the local units can be remembered or predicted better with stimuli that are structurally redundant (i.e., good patterns) than with stimuli that are not (i.e., poor patterns). All of these studies have used stimuli such as dot patterns that differ only globally in the relative location of the local units (i.e., not in the number of absolute locations). The question remains as to whether such relative location information can actually be used to facilitate local processing.

\section{Local Processing: Identification and Integration of Local Features}

That location information is used at all during local processing has been established by various researchers. Feature-integration theory (Treisman \& Gelade, 1980; Treisman, Sykes, \& Gelade, 1977) and related research (Prinzmetal, 1981; Prinzmetal \& Millis-Wright, 1984) indicate that individual features of local units in a display can be identified without association to any spatial location, but proper integration of those features into local units requires some kind of information about spatial location.

Treisman (e.g., Treisman et al., 1977) has shown that spatial information about features of local units is not initially encoded during feature identification. For example, Treisman and Schmidt (1982) required subjects to report all the colored letters in a briefly presented display. They found that subjects often produced conjunction errors: Colors and letters present in the display were reported in combinations that did not actually occur. When these conjunction errors were made at a higher rate than conjunctions of a feature that was absent from the display with one that was present (called feature errors), the excess rate of conjunction errors was thought to reflect $i l-$ lusory conjunctions. These illusory conjunctions were attributed to perceived features that were incorrectly located. The occurrence of illusory conjunctions strongly supports the contention that spatial information about features of local units is not initially specified. According to Treisman's theory, after the initial identification of features, a perceiver then focuses attention on one spatial location after another in the visual array in order to determine which features belong to which particular object for proper integration. This claim is based on findings that individual features can be identified in parallel but identification of conjunctions involves serial processing (Treisman \& Gelade, 1980).

Although Treisman and her colleagues have shown that specific spatial location is not initially encoded during feature identification, they have not directly examined the nature of the spatial location information that is used during feature integration. Prinzmetal (1981; Prinzmetal \& Millis-Wright, 1984) has shown that some global information about spatial location can influence feature integration. For example, Prinzmetal (1981) briefly presented

stimulus arrays that consisted of two rows of circles. (See Figure 1 for sample stimuli.) In some of the arrays, two circles each contained a single vertical or horizontal line segment. In other arrays, one circle contained a single line segment and another a plus sign. The two encircled items could occur in the same row or in different rows. The subject's task on each trial was to determine whether or not a plus sign had occurred. The results from this experiment showed that illusory conjunctions (i.e., reporting a plus sign when a horizontal segment occurs in one circle and a vertical segment in another at a higher rate than guessing) were more likely to occur if both items were contained by the same row rather than by different rows. This finding was obtained even when the distance between the circles was held constant. Further, the rate of feature errors was as high within groups as between groups. This work indicates that the group to which individual features belong affects whether or not they will be conjoined into local units; consistent with Treisman's theory, such global location information (i.e., segregation into groups) did not affect individual feature identification. Prinzmetal and Millis-Wright (1984) suggest that more illusory conjunctions occur within groups because focal attention is not directed internally to the distinct properties of units within the group.

In Prinzmetal's (1981) study, more illusory conjunctions occurred within a perceived group of objects that are not globally segregated from each other than across perceived groups. Yet research on pattern goodness suggests that grouping is not simply present or absent. These studies (e.g., Bear, 1973; Bell \& Handel, 1976) have shown that global patterns can differ in the degree to which they organize and in how well they can specify the relative location of local units. Global patterns that are simple or redundant in structure are processed more quickly and accurately than others in information-processing tasks (e.g., Garner \& Sutliff, 1974; Sebrechts \& Garner, 1981). The more redundant the pattern, the faster and more accurate the processing.

\section{Purpose of the Present Study}

In the present study, we exploited the phenomena of illusory conjunctions and pattern goodness in order to de-

(a)

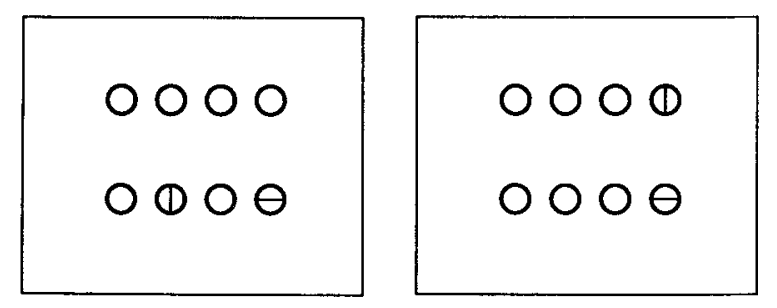

Figure 1. Sample stimuli used in Prinzmetal's study. In panel a, items occur in the same row and in panel $b$, items occur in different rows (adapted from Prinzmetal, 1981). 
termine whether or not global patterns can provide information about where the local units are located above and beyond segregation from the rest of the visual field. We examined whether information about the relative location of local units can facilitate their identification to a greater degree within groups that specify relative location better. Good and poor global groupings were used in a local target-detection task similar to Prinzmetal's (1981). Subjects were asked to determine whether a local conjunction (e.g., a plus sign) occurred in each array. Note that global information was irrelevant to task performance. If global information affects performance, then we can conclude that it affected local processing.

The present methodology, which includes accuracy measures of both local feature identification and integration, allows us to distinguish the proposed global input to local processing from the possibilities that (1) the global level is simply processed prior to the local in a sequential fashion, and (2) a salient global unit draws focal attention away from local feature integration. The first possibility is a straightforward global-precedence situation. The second possibility was suggested by Prinzmetal and MillisWright (1984) and derives from Treisman and Gelade's (1980) findings that focal attention is required for identifying conjunctions but not individual features.

Variation of pattern goodness. The particular instantiation of pattern goodness used in this study was based on the oblique effect-the finding that performance with obliquely aligned stimuli is slower and less accurate than that with vertically or horizontally aligned stimuli (e.g. , Essock, 1980). Lasaga and Garner (1983) have shown that vertical and horizontal lines are more easily encoded and that oblique lines are more confusable with each other. The confusability of the diagonals indicates that the specific relative location of their endpoints is not well registered.

The oblique effect is a robust phenomenon that lends itself well to an examination of the effects of global goodness on feature integration. We can use simple arrays consisting of only two distinct locations in order to examine performance with their local units. (See also Cecala \& Garner, 1986, for evidence that even a single dot in a frame can imply orientation.) In this way, we can avoid the possibility of unidentified subparts, which exists with the 5-dot patterns typically used to study pattern goodness.

Predictions for global goodness. Given that our task requires only local information, if global goodness has an effect on local target detection, then it has interfered with or facilitated local processing. The pattern of results will help us to distinguish between the types of global influence at play. If relative location is actually provided as input to the local level during feature integration, then variations in global goodness should affect the accuracy with which local features are integrated but not identified. That is, good patterns should facilitate local feature integration more than poor patterns, because good pat- terns can specify relative location better. On the other hand, if a salient global unit draws focal attention away from local processing, then it should affect feature integration only (Treisman \& Gelade, 1980) in an opposite manner; a more salient global property should produce a higher rate of illusory conjunctions. Sebrechts and Fragala (1985) have shown that a good global pattern is more salient than a poor one and tends to interfere more with overall identification of local patterns. Thus, a good global pattern should draw resources away from local feature integration more than a poor one. Finally, if the local and global levels are simply processed sequentially with the global level completed first, then a global property that is processed prior to the local should detract from both the identification of local features and their integration into local units, in the same way.

Pattern goodness, spacing, and global precedence. An important stimulus aspect that has been found to affect the salience of global properties is their density (i.e., the spacing between objects of a group). Martin (1979) found that with high-density stimuli, global information was processed more quickly than local, and with lowdensity stimuli, local information was processed more quickly. Such a manipulation was used in the present study to influence the salience of the global properties in order to better elucidate their effects on local processing, if any.

Variation of spacing. The objects in each array were presented under small and large spacing. Globally, spacing affects the density of the global pattern. Locally, spacing reflects absolute distance between items. ${ }^{1}$

Predictions about spacing. Of particular interest will be any interaction of spacing with global goodness. The effects of pattern goodness should decrease in strength from small to large spacing. No main effect of spacing is expected on illusory conjunctions (which would simply reflect an effect of absolute distance). Treisman and Schmidt (1982) found little evidence for any distance effect on illusory conjunctions, and Prinzmetal and MillisWright (1984) obtained unclear results. A main effect of spacing on feature errors may possibly occur only in the sense that local units with large spacing between them are more peripheral than those with small spacing, requiring more energy for registration (Weynmouth, 1958).

\section{EXPERIMENT 1}

In Experiment 1, we examined the effects of global goodness on the rate of illusory conjunctions (i.e., errors in local feature integration) and the rate of feature errors (i.e., errors in local feature identification). The individual local features used in this experiment were a vertical and a horizontal line segment.

\section{Method}

Subjects. Twenty undergraduates at the University of Virginia participated as subjects in order to fulfill a course requirement. All 
participants had normal or corrected-to-normal vision. Each subject participated in a $1-\mathrm{h}$ session.

Stimuli. The stimuli were created with an Apple Macintosh computer and printed with an Apple LaserWriter. The figures were pasted onto cards for presentation on the tachistoscope. Each stimulus contained only two encircled items. Either both items were simple features (vertical or horizontal line segments) or one was a simple feature and the other a conjunction of features (a plus sign). (See Figure 2 for sample stimuli.) The diameters of the circles in each stimulus subtended a visual angle of $0.36^{\circ}$. The background luminance was set at $17.1 \mathrm{~cd} / \mathrm{m}^{2}$.

There were five possible pairs of items that the circles contained: two horizontal line segments (HH); two vertical line segments (VV); a vertical and a horizontal line ( $\mathrm{VH}$ ); a plus sign and a horizontal line (PH); and a plus sign and a vertical line (PV). Each of the stimuli in which no plus sign actually occurred (VH, HH, VV) was presented twice as often as each of the stimuli with a plus sign present. When the items in an array were different, each item was presented equally often in each circle.

The two circles in each stimulus were aligned in one of four ways: along a vertical axis, a horizontal axis, a positive diagonal axis (with $+45^{\circ}$ slope), or a negative diagonal axis (with $-45^{\circ}$ slope). (See Figure 3.) The vertical and horizontal axes were considered good arrangements and will be referred to as main axes. The diagonal axes were considered poor arrangements and will be referred to as oblique axes.

Each of these arrangements was presented under two conditions of spacing: small (subtending $3^{\circ}$ visual angle) and large (subtending $5^{\circ}$ visual angle). (See Figure 3.) The size of the circles was not changed across spacing conditions.

A master set of 94 stimuli was created for the experimental trials, as follows. The two elements in the target-present stimuli (PH, PV) were presented in each of the four global arrangements and in each of the two spacing conditions, with each element presented in each of the two circles. These combinations produced $16 \mathrm{PH}$ trials and 16 PV trials. Thus, the master set contained 32 target-present stimuli. The two elements in the target-absent stimuli (VV, HH, VH) were also presented in each of the four global arrangements and under each of the two spacing conditions. Further, the two VH elements occurred equally often in each of the two circles. Thus, there were $8 \mathrm{VV}$ stimuli, $8 \mathrm{HH}$ stimuli, and $16 \mathrm{VH}$ trials.

Procedure. The procedure was adapted from Prinzmetal's (1981) study. Before each trial, the experimenter gave a "ready" signal, and the subject initiated the presentation of the stimulus by pressing a button held in the left hand. The stimulus was then presented in a three-channel, Scientific Prototype (Model GB) tachistoscope,

\begin{tabular}{|c|c|c|}
\hline $\begin{array}{l}\text { Vert Line/Vert Line: } \\
\text { (Horiz Line/Horiz Line) }\end{array}$ & $\begin{array}{l}\text { (1) } \\
\text { (1) }\end{array}$ & $\begin{array}{c}\text { TARGET ABSENT } \\
\text { Same Feotures } \\
\text { (VV, HH) }\end{array}$ \\
\hline Vert Line/Horiz Line: & $\begin{array}{l}\Phi \\
\theta\end{array}$ & $\begin{array}{l}\text { TARGET ABSENT } \\
\text { Different Feotures } \\
(\mathrm{VH})\end{array}$ \\
\hline $\begin{array}{l}\text { Plus/Vert Line: } \\
\text { (Plus/Horiz Line) }\end{array}$ & $\begin{array}{l}\oplus \\
1\end{array}$ & $\begin{array}{c}\text { TARGET PRESENT } \\
(\mathrm{PV}, \mathrm{PH})\end{array}$ \\
\hline
\end{tabular}

Figure 2. Examples of feature combinations used in Experiment 1. The top two frames illustrate target-absent stimuli with same features and with different features, respectively; the bottom frame illustrates the target-present feature combinations.

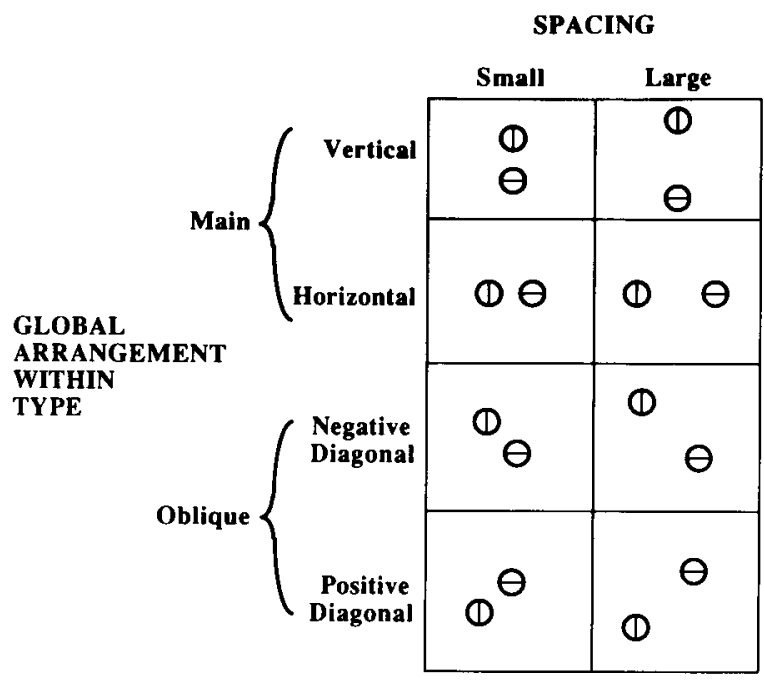

Figure 3. Manipulations of spacing and type of global arrangement used in Experiment 1. The vertical and horizontal arrangements were considered to be good arrangements and the oblique arrangements were considered to be poor, on the basis of the oblique effect.

followed by a regular mask of black dots. The mask was also present between trials. The subject was instructed to look at the center of the mask but spread his/her attention to the entire region before each trial. The stimulus and mask were viewed binocularly from a distance of $127 \mathrm{~cm}$. The subject's head was restrained from movement by an eyepiece that did not allow head rotation. The subject's task was to determine whether or not a plus sign had occurred. The subject pushed a lever in one direction if the target was present in the stimulus and in the opposite direction if it was not. The response and its accuracy were recorded by an Apple IIe computer.

At the beginning of the session, the subjects were told that about one half of the stimuli would contain the target, even though only one third of the trials actually contained a plus sign (see Prinzmetal, 1981). The subjects were instructed in this fashion in order to encourage them to adopt a neutral response criterion.

For the experimental trials, the master set of stimuli was randomized for each subject, with the constraint that one stimulus from each cell in the design (see Figure 3) occur in each block of 8 trials. The order of trials within each block was completely random. This set of stimuli was then presented to the subject 4 times, alternating between forward and reverse order. Before the experimental trials, each subject performed 48 practice trials, which were chosen randomly such that the number of each type of stimulus was proportional to its frequency in the master set.

During practice trials, the exposure duration was gradually adjusted with a modified staircase procedure until the average accuracy rate for a block of eight trials reached $70 \%$. During the experimental trials, the average accuracy rate continued to be monitored after each block of eight trials and exposure duration modified, if necessary, in order to maintain performance at this level. Exposure duration was decreased by $5 \mathrm{msec}$ if accuracy was above $70 \%$, and exposure duration was increased by $5 \mathrm{msec}$ if accuracy was below $70 \%$. The mean exposure duration for all subjects throughout the experimental session was $49 \mathrm{msec}$ (the mean standard deviation was $8 \mathrm{msec}$ ).

\section{Results}

Responses of interest were those in which a plus sign was reported as present when it actually did not occur 
(i.e., errors on target-absent trials with same features and with different features). ${ }^{2}$ Since a criterion was set for the proportion of errors that a subject made in the session, the number of incorrect target-present trials and the number of incorrect same-feature and different-feature targetabsent trials together must all sum to approximately $30 \%$ of the total number of trials. Thus, these proportions are not independent. However, the proportion of errors on same-feature trials is free to vary relative to that on different-feature trials. Performance on both could be equally accurate or not. Note also that we will be comparing the effects of global goodness and spacing on the proportion of error for each type of single-feature trial. So, if subjects made errors on every single differentfeature trial, then they would have made $100 \%$ error on these trials, etc.

In all analyses reported below, the factor of individual global arrangement (vertical, horizontal, positive and negative diagonal) was always included and nested within type of global arrangement (main, oblique). However, no significant effect of individual global arrangement was ever obtained. To simplify the report of the findings, the lack of significance of this factor will not be mentioned further.

Feature errors. First, errors on same-feature trials (VV, HH) were analyzed. Because correct performance on these trials required identification of both features but not information about their spatial location, the error rates will tell us whether any global properties influenced the identification of local features. On these trials, both features necessary for a plus sign were not present; thus, er- rors on these trials (i.e., reporting a conjunction when none occurred) reflect pure guessing (when no stimulus information was processed) and/or sophisticated guessing (when some stimulus information about individual features was processed but not all; see Broadbent, 1967).

The percent errors for same-feature stimuli were submitted to a three-way analysis of variance (ANOVA) of spacing (small, large), global axis (vertical, horizontal, negative and positive diagonal), and type of global arrangement (main, oblique) in which global axis was nested. (See Figure 4 for the mean percent error in responses to same-feature stimuli, according to type of arrangement and spacing.) The results of this analysis revealed only a significant effect of spacing $[F(1,19)=$ $38.79, p<.01]$. Stimuli with large spacing between items produced a higher guessing rate $(M=40 \%)$ than did stimuli with small spacing $(M=24.05 \%)$.

Illusory conjunctions. The rate of errors on differentfeature trials can reflect guesses (pure and/or sophisticated) and errors due to perceptual mislocation of actually registered features (i.e., the vertical and horizontal lines). Thus, the likelihood of illusory conjunctions needs to be estimated by comparing performance on differentfeature trials with that on same-feature trials. For each subject, this estimated percentage of illusory conjunctions (est- $P_{\text {Iconjunction }}$ ) was calculated as follows:

$$
\text { est- } P_{\text {Iconjunction.cell }}=P_{\text {different.cell }}-P_{\text {same.cell }},
$$

where est- $P_{\text {Iconjunction.cell }}$ is the estimated percentage of different-feature errors that are illusory conjunctions; $P_{\text {different.cell }}$ is the total percentage of different-feature er-

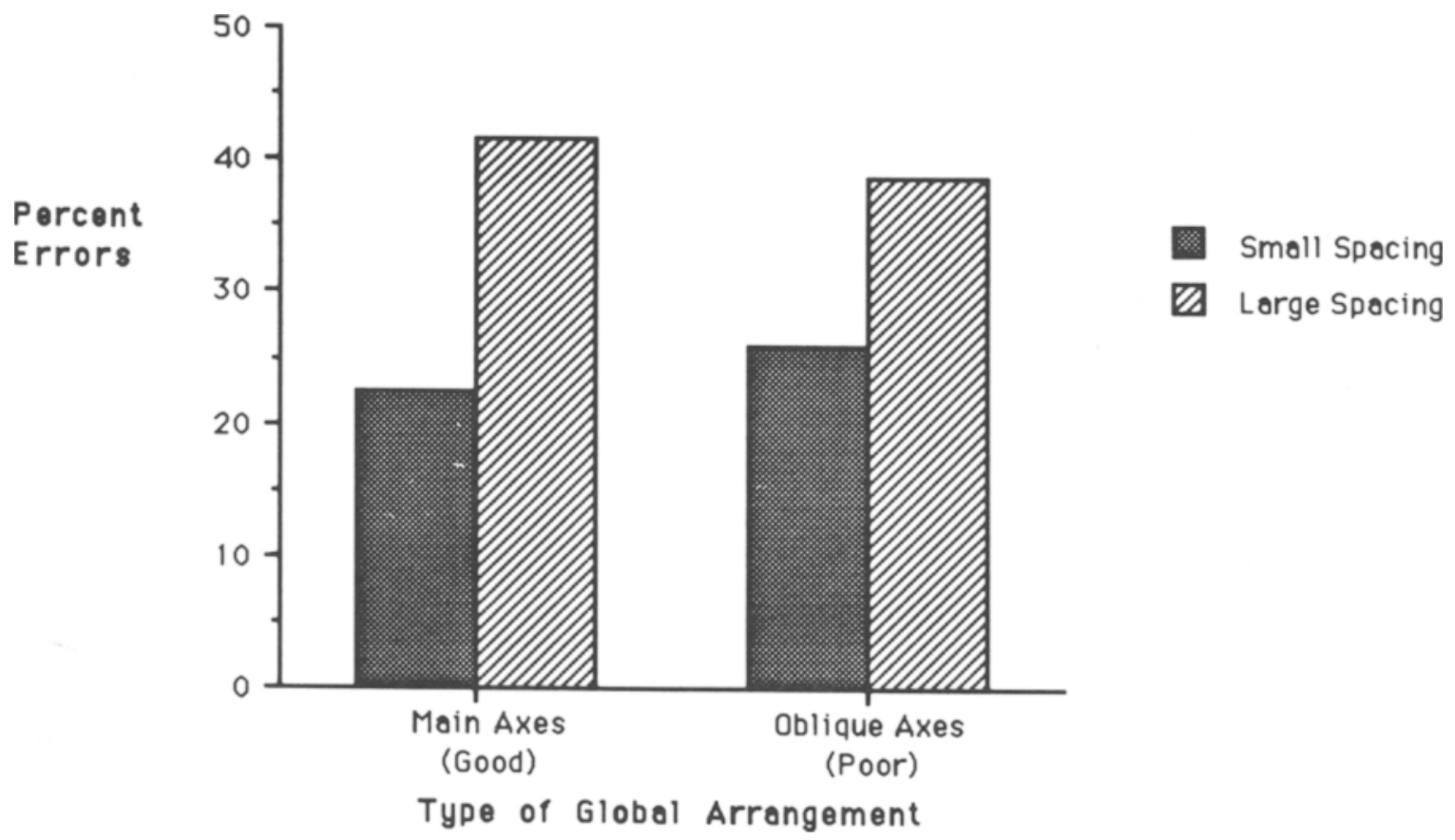

Figure 4. Mean percent error on same-feature trials according to spacing and type of global arrangement (Experiment 1, using vertical and horizontal line segments). 
rors for that cell in the design; and $P_{\text {same.cell }}$ is the percentage of same-feature errors (i.e., the baseline rate of guessing) for that cell in the design. If est- $P_{\text {Iconjunction }}$ is equivalent to zero, then having the two different features of a conjunction actually present though not conjoined physically was not more likely to produce an error than having two identical features that could not be conjoined into a plus sign. It will be important to first establish whether the various rates of illusory conjunctions differ from zero. Note that any effect that the global properties may have had, perceptually, in inducing a "targetpresent" response when the two local features of a conjunction were not physically present is being removed from the rates of illusory conjunctions in each cell. This includes any interactions between the identity of the global unit with the identity of the local unit, which are not of interest in this paper.

By using the error rate on same-feature trials for each cell in the design (i.e., for each combination of spacing and individual global arrangement within type of global arrangement), we make no assumptions about whether any of the experimental factors or their combinations produced differential (pure and/or sophisticated) guessing rates for any individual subject. Figure 5 shows the estimated percent conjunction errors across subjects as a function of type of global arrangement and spacing.

First the average est- $P_{\text {Iconjunction }}$ in each cell was tested to determine whether it differed significantly from zero.
For each subject, est- $P_{\text {Iconjunction }}$ for each of the two global axes nested within each type of global arrangement was included separately so that no assumptions would be made about their equivalence. The average rate of illusory conjunctions was significantly different from zero for both main and oblique arrangements under conditions of small spacing [for main axes, $t(39)=5.01, p<.05$, and for oblique axes, $t(39)=2.36, p<.05]$. When spacing was large, only the average rate of illusory conjunctions for main axes was significantly different from zero [for main axes, $t(39)=2.07, p<.05$, and for oblique axes, $t(39)<1$, n.s.]. Thus, when spacing was large, type of global arrangement had an effect in the sense that the rate of illusory conjunctions for main axes exceeded zero, whereas that for oblique axes did not.

Next, the estimated percents of illusory conjunctions were submitted to a three-way ANOVA of global axis nested within type of global arrangement by spacing. (Although one cell was no different from zero, it was important to determine whether there was a main effect of type of global arrangement or of spacing, or any interaction between the two variables.) The results revealed a significant effect of type of global arrangement. The main axes produced more illusory conjunctions $(M=10.31 \%)$ than oblique axes $[M=3.98 \% ; F(1,19)=7.43$, $p<.01]$. The effect of spacing was also significant. A higher percentage of illusory conjunctions $(M=11.3 \%)$ was obtained when spacing was small than was obtained

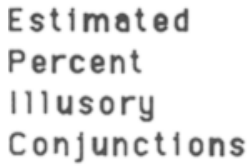

Estimated
Percent
Illusory
Conjunctions

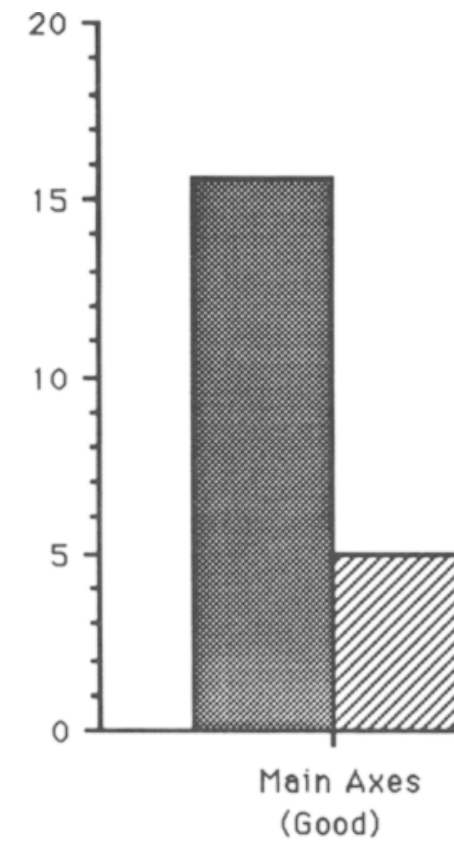

Small Spacing Large Spacing

\section{Type of Global Arrangement}

Figure 5. Mean estimated percent illusory conjunctions as a function of spacing and type of giobal arrangement (Experiment 1 , using vertical and horizontal line segments). 
when spacing was large $[M=2.97 \% ; F(1,19)=8.51$, $p<.01]$. The interaction between spacing and type of global arrangement was not significant $(F<1)$. In sum, subjects were more likely to produce illusory conjunctions in main rather than oblique arrangements and with small rather than large spacing between the features.

\section{Discussion}

First, the pattern of results obtained for feature errors (full or partial guessing behavior) as a function of spacing instructs us on how to interpret the pattern obtained for illusory conjunctions: Subjects produced fewer feature errors and more illusory conjunctions with small spacing between items than with large spacing. Such results, taken together, suggest that we may be misinterpreting the meaning of illusory conjunctions. Although illusory conjunctions do not involve completely accurate extraction of information from the visual array, these errors reflect the extraction of more correct information (i.e., the identification of both features, though not their spatial location) than when a subject resorts to pure or partial guessing. A lower rate of feature errors coupled with a higher rate of illusory conjunctions for a condition may mean that subjects were able to extract more stimulus information rather than less when they committed an illusory conjunction; and the more illusory conjunctions, the more stimulus information extracted more often.

Since large and small spacing occurred with equal probability, and since subjects were instructed to spread their attention across the mask area between trials, the present findings obtained for spacing can best be explained in terms of retinal eccentricity of the individual features: Given the limited time to register local information, the more eccentric the information (i.e., large spacing between items), the more energy required for detection and identification (Weynmouth, 1958).

No effect of type of global arrangement (i.e., main vs. oblique) on the rate of feature errors was obtained, whereas an effect was found on illusory conjunctions. Oblique arrangements produced fewer illusory conjunctions than main ones. This pattern of results agrees with the predictions of the hypothesis that salient global properties detract focal attention from local feature integration.

\section{EXPERIMENT 2}

Based on comments made by a couple of subjects in Experiment 1, we closely examined our stimulus set again and suspected a possible structural factor that could also account for the effects of pattern goodness in that experiment. Notice, in Figure 3, that for vertical and horizontal arrangements, the features seem to translate easily toward each other when you try to image a conjunction between them, whereas those in the oblique arrangements do not. Perhaps the larger percentage of illusory conjunctions obtained for the vertical and horizontal arrangements in Experiment 1 was due to the fact that their features could be translated more easily to produce plus signs, given their relative locations. In contrast, the diagonal fea- (a)

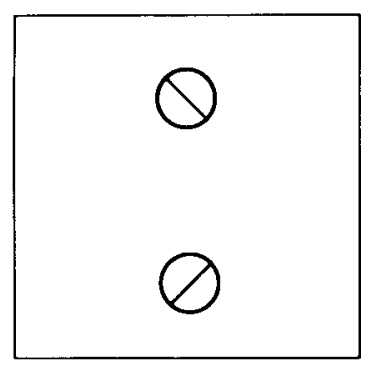

Figure 6. Sample stimuli used in Experiment 2, in which the line segments are diagonal. In panel $a$, items are vertically aligned and seem to translate less easily towards each other than in pand b, where the items are obliquely aligned.

tures in Figure 6b seem to translate more easily toward each other than those in Figure 6a. This notion of translatability is consonant with that of good continuation and that of spatial relatability (Kellman \& Loukides, 1987; Kellman \& Shipley, 1990). Spatial relatability is a term that has been used to refer to the joining of spatially separated edges such that the two meet with no discontinuity between them. Since we are using simple encircled line segments, and we do not know whether any occlusions (typically assumed for good continuation), edges, or surfaces are being perceived, we will use the general geometric term, translatability, to refer to the phenomenon. No assumption is made about whether the phenomenon differs from the notions of good continuation or relatability.

In Experiment 2, we examine this alternative explanation of translatability by using diagonals as the simple local features. If global goodness detracts focal attention from local feature integration, then we should replicate Experiment 1. If ease of translation is at play, then illusory conjunctions should now be more likely within the oblique axes.

\section{Method}

Subjects. Twenty undergraduates at the University of Virginia participated in this experiment for course credit. All had normal or corrected-to-normal vision. None of the subjects had participated in Experiment 1.

Stimuli. The stimuli were constructed as in Experiment 1. However, instead of using local horizontal and vertical features, positive diagonals $\left(+45^{\circ}\right.$ from vertical) and negative diagonals $\left(-45^{\circ}\right.$ from vertical) were used (see the examples in Figure 6). Instead of a plus sign, target-present trials contained an "X."

Procedure. The procedure was the same as in Experiment 1, except that the task was to determine whether or not an " $\mathrm{X}$ " was present in the display. The mean exposure duration for all subjects throughout the experimental session was 49 msec (the mean standard deviation was $11 \mathrm{msec}$ ).

\section{Results}

For all analyses reported below, the factor of individual global axis was nested within type of global arrangement, but no significant effect was ever obtained. 
Feature errors. Mean percent error on same-feature trials by type of global arrangement and spacing are shown in Figure 7. Again, spacing influenced the rate of guessing $[F(1,19)=21.88, p<.01]$. With large spacing, guessing occurred more often $(M=37.16 \%)$ than with small $(M=25.08 \%)$. As in Experiment 1, subjects were unable to extract as much feature information when items were far apart than when they were close together. The identity of local information in tighter clusters is still being processed more accurately. As in Experiment 1, the variation of global goodness did not influence the accuracy of feature identification in these same-feature trials.

Illusory conjunctions. The percent illusory conjunctions obtained for each cell was estimated using the same equation as in Experiment 1. (Average percent illusory conjunctions as a function of spacing and type of global arrangement are shown in Figure 8.) Again, these estimated percents were first tested to determine if they differed from zero. The rate of illusory conjunctions was significantly different from zero for both types of arrangement under conditions of small spacing [for main axes, $t(39)=6.52, p<.05$; for oblique axes, $t(39)=3.04$, $p<.05]$. With large spacing between the items, neither of the rates differed significantly from zero [for main axes, $t(39)<1$, n.s.; for oblique axes, $t(39)=1.27$, n.s.]. Regardless of the type of global arrangement, spacing again had an effect in the sense that the rate of illusory conjunctions for arrays with small spacing exceeded zero whereas that for arrays with large spacing did not.

The percents of illusory conjunctions for each cell by subject were submitted to a three-way ANOVA of in- dividual global axis within type of global arrangement by spacing. Spacing influenced performance in the same way as in Experiment 1. With small spacing between items, more illusory conjunctions $(M=13.75 \%)$ were obtained than with large spacing $[M=3.67 \% ; F(1,19)=9.48$, $p<.01]$. The results also revealed that type of global arrangement mattered, but that more illusory conjunctions occurred with oblique arrangements $(M=11.68 \%)$ than with main $[M=5.78 \% ; F(1,19)=4.37, p<.0502]$. This pattern is completely opposite to that obtained in Experiment 1 .

Finally, there was a significant interaction between type of global arrangement and spacing $[F(1,19)=4.38$, $p<.05$ ]. Oblique arrangements produced more illusory conjunctions $(M=19.5 \%)$ than main arrangements ( $M=7.97 \%$ ) when the spacing between items was small ( $p<.05$; Newman-Keuls test); but when the spacing was large, there was no difference between the percent of illusory conjunctions produced for oblique $(M=3.75 \%)$ and for main $(M=3.59 \%)$ global arrangements (n.s.; Newman-Keuls test).

\section{Discussion}

The effect of spacing was the same as in Experiment 1. These effects are again best attributed to the distance of the local features from the fovea.

Type of global arrangement again only had an effect on illusory conjunctions. However, the pattern of results was reversed from that of Experiment 1. Mllusory conjunctions of diagonal line segments arose more often in diagonal arrangements, in which the line segments could

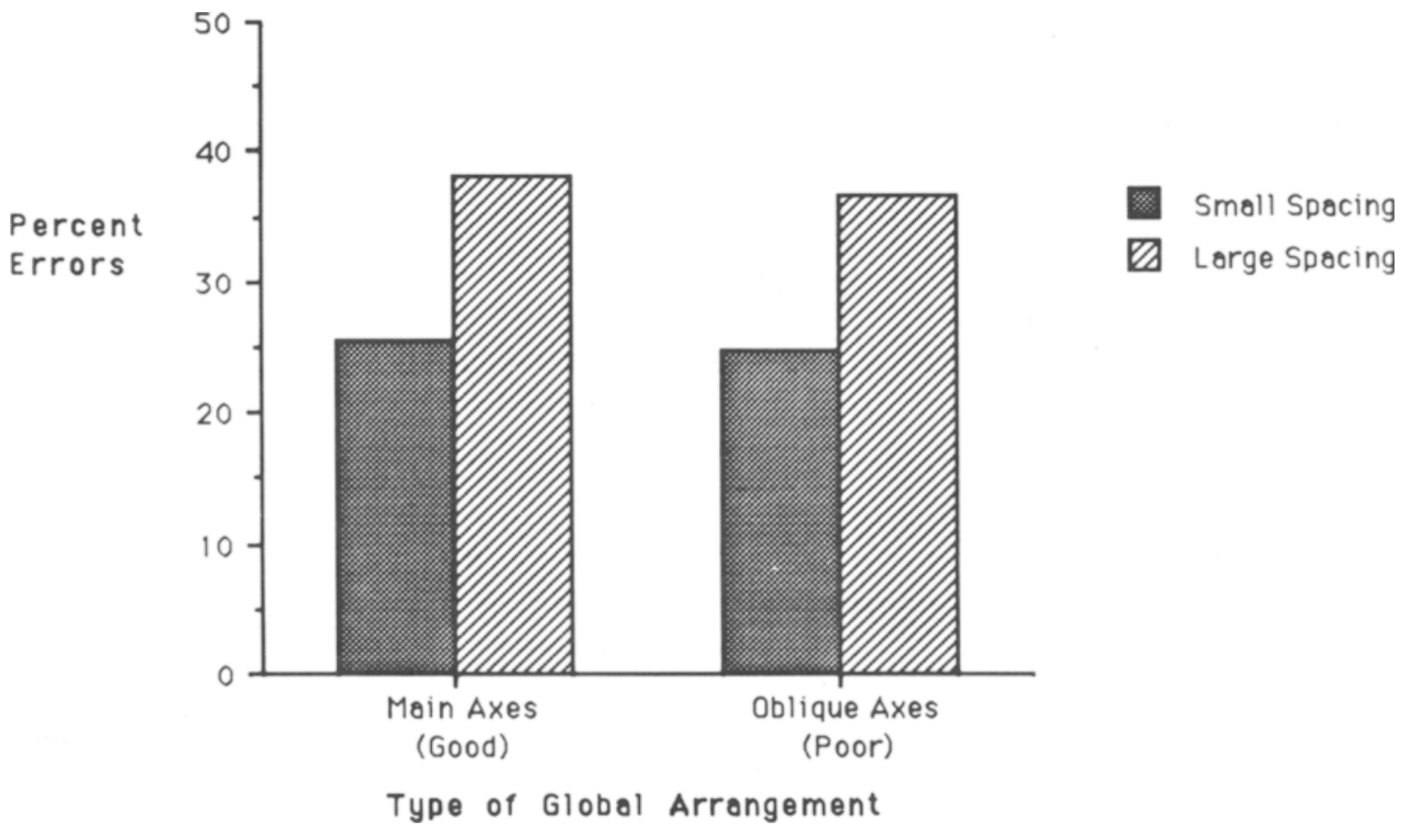

Figure 7. Mean percent error on same-feature trials according to spacing and type of global arrangement (Experiment 2, using diagonal line segments). 


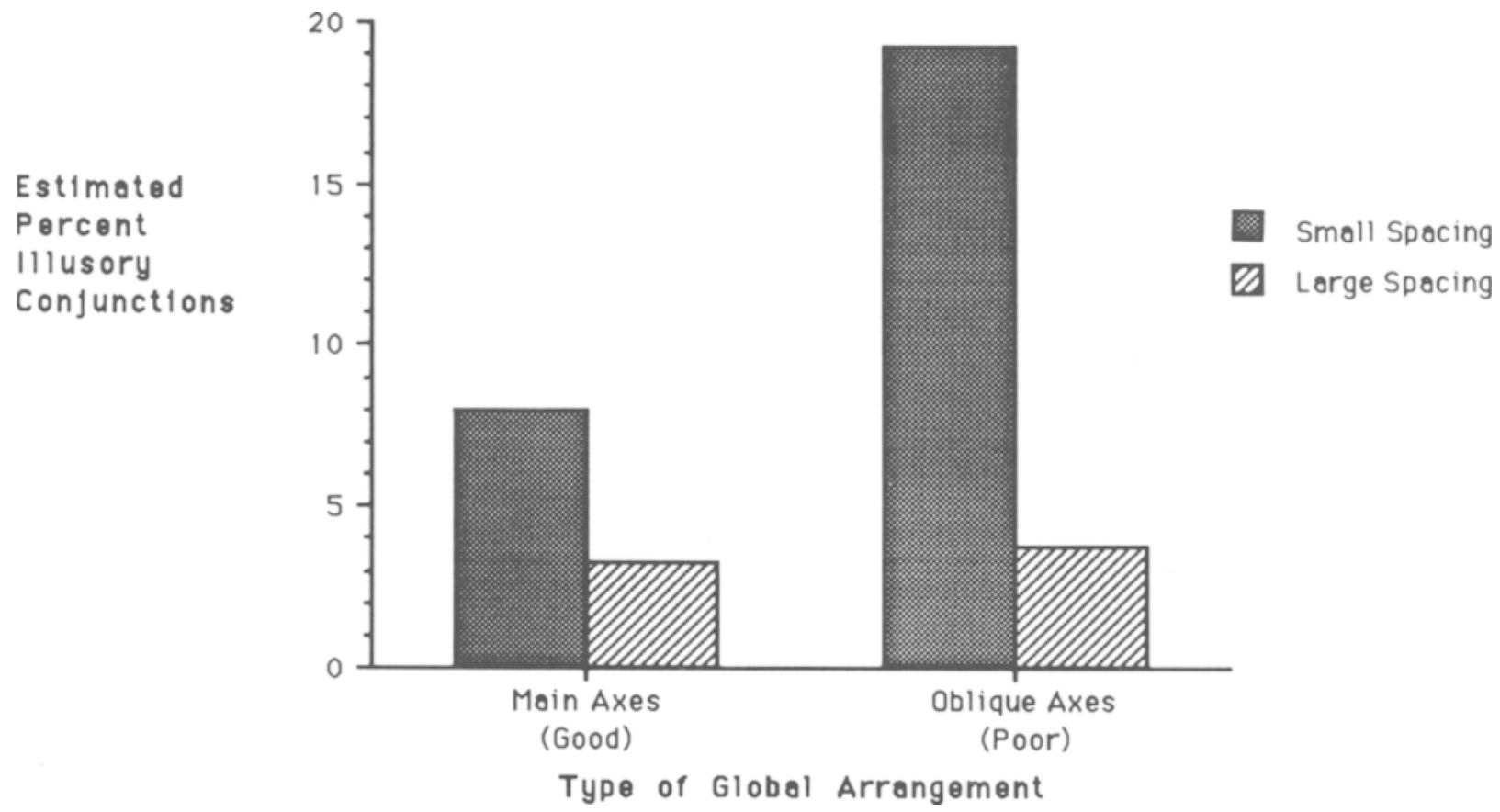

Figure 8. Mean estimated percent illusory conjunctions as a function of spacing and type of global arrangement (Experiment 2, using diagonal line segments).

be more easily translated (displaced) toward each other. Thus, the originally intended manipulation of global goodness had no direct effect on performance.

The interaction between type of global arrangement and spacing obtained with illusory conjunctions also seems to be due to greater sensory limitation with large spacing. The rates of illusory conjunctions were no different from zero with large spacing, and high rates of feature errors were also obtained with large spacing.

\section{GENERAL DISCUSSION}

In both experiments, spacing consistently affected performance in the same way. Smaller spacing produced fewer feature errors and more illusory conjunctions. Both of these patterns, taken together, indicate that if some but not all information is extracted from an array, it tends more often to be the identity of the individual features without their spatial location. This pattern also reveals that a greater rate of illusory conjunctions reflects the extraction of more stimulus information rather than less. Thus, it is important to take both feature errors and illusory conjunctions into account. Since large spacing between items produced many guesses and few illusory conjunctions, the effect of spacing in both experiments was best explained in terms of a greater sensory limitation when items occurred farther from the fovea.

The results from Experiment 1 for global goodness supported the hypothesis that good patterns detract focal attention from local feature integration (but not from local feature identification, for which attention is not required). Type of global arrangement had no effect on the production of feature errors, yet illusory conjunctions were more likely to occur with good global arrangements than with poor arrangements.

The results from Experiment 2 clearly showed that when the local features are changed to diagonal line segments, more illusory conjunctions arise when the segments are arranged obliquely than when they are arranged horizontally or vertically. This reversal in pattern of results was strong when the local features were close together, but the difference was nonsignificant when the features were far apart.

The results from Experiments 1 and 2 taken together indicate that the main factor influencing the production of illusory conjunctions with main and oblique arrangements was not the goodness of the arrangement, per se, but the ease with which the line segments intersect when translated (i.e., displaced in the direction of their extent). That is, features that are not yet correctly located in space are more likely to be displaced toward each other, perceptually, in the direction of their extent. When a positive and a negative diagonal line segment are placed diagonally from each other, they are more likely to conjoin as an illusory " $X$ " than when they are placed vertically or horizontally from each other. When a horizontal and a vertical line segment are placed vertically or horizontally from each other, they are more likely to conjoin as an illusory " +" than when they are placed diagonally from each other. If the line segments had simply been 
registered without any information about their spatial location and without any constraint on mislocation, then there should have been no difference between the types of arrangement, regardless of the specific pair of features used. Illusory conjunctions, if any, should have been produced randomly. If the perceptual error was to bring information in the visual field closer together, then again there should have been no difference due to type of arrangement or type of features. If the perceptual error was to bring information in a group closer together, the effect should have been stronger for the vertical and horizontal arrangements than for the oblique arrangements, but the specific pair of features should not have made any difference and a reversal in pattern as a function of global goodness certainly should not have occurred. The present results strongly suggest some type of perceptual constraint in which line segments are more likely to be mislocated in the direction of their extent, rather than randomly or as a function of absolute distance.

There are several implications of these findings. First, they indicate that such a factor should not be ignored in future examinations of global influence on local feature identification and integration. Second, ease of conjunction due to segment translation may have influenced results in similar studies in the past. The translatability of line segments seems to have been an artifact in Prinzmetal's (1981) Experiment 2, but not in his other experiments. Finally, the finding that the rate of illusory conjunctions depends on the nature of the features and how they are arranged reveals that the system does pick up some information about spatial location before focused attention is applied.

It should be noted that the results of type of global arrangement obtained for illusory conjunctions cannot be attributed to any similarity relationship between the local and global levels. Whatever similarity relations exist between the global axes and the local features, if any are at play, they will affect performance with the same-feature stimuli. By subtracting the effects of type of global arrangement on these trials from the effects of type of global arrangement on different-feature trials, we remove any local/global similarity effects from the estimated rates of illusory conjunctions.

Although we did not find evidence to support any of the three models of global influence that we set out to test, the question of the nature of global influence remains an important one and merits further investigation. The oblique effect, our particular instantiation of global goodness, is a robust effect, and it provided a strong manipulation of goodness. The visual angles that we used were well within the range that produces faster global identification rather than local identification of patterns (Kinchla $\&$ Wolfe, 1979). Our stimuli were simple rotations of the exact same physical configuration. Yet, although vertical and horizontal axes are encoded more easily and the relative direction of their endpoints is specified better compared with oblique patterns, vertical and horizontal arrangements did not help their local units to be encoded better spatially. Nor did our instantiation of global goodness produce any evidence of simple global precedence or of detraction of the global unit from local feature integration. Albeit a null result, these findings suggest that global processing may not facilitate or interfere with local processing of single line segments in the visual field.

\section{REFERENCES}

Attneave, F. (1955). Symmetry, information, and memory for patterns. American Journal of Psychology, 68, 209-222.

BEAR, G. (1973). Figural goodness and the predictability of figural elements. Perception \& Psychophysics, 13, 32-40.

BeLL, H. H., Handel, S. (1976). The role of pattern goodness in the reproduction of backward masked patterns. Joumal of Experimental Psychology: Human Perception \& Performance, 2, 139-150.

BRoADBENT, D. E. (1967). Word-frequency effect and response bias. Psychological Review, 74, 1-15.

Cecala, A. J., \& Garner, W. R. (1986). Internal frame of reference as a determinant of the oblique effect. Journal of Experimental Psychology: Human Perception \& Performance, 12, 314-323.

Essock, E. A. (1980). The oblique effect of stimulus identification considered with respect to two classes of oblique effects. Perception, 9, $37-46$.

GARNER, W. R., SUTLIFF, D. (1974). The effect of goodness on encoding time in visual pattern discrimination. Perception \& Psychophysics, 16, 426-430.

Kellman, P. J., \& Loukides, M. G. (1987). An object perception approach to static and kinetic subjective contours. In S. Petry \& G. E. Meyer (Eds.), The perception of illusory contours (pp. 151-164). New York: Springer.

Kellman, P. J., \& Shipley, T. F. (1990). Visual interpolation in object perception: A computational theory. Unpublished manuscript.

KINCHLA, R. A., \& WolFE, J. M. (1979). The order of visual processing: "Top-down," "bottom-up," or "middle-out." Perception \& Psychophysics, 25, 225-231.

LASAGA, M. I. (1989). Gestalts and their components: Nature of information precedence. In B. E. Shepp \& S. Ballesteros (Eds.), Object perception: Structure \& process (pp. 165-202). Hillsdale, NJ: Erlbaum.

Lasaga, M. I., \& Garner, W. R. (1983). The effect of line orientation on various information processing tasks. Journal of Experimental Psychology: Human Perception \& Performance, 9, 215-225.

MARTIN, M. (1979). Local and global processing: The role of sparsity. Memory \& Cognition, 7, 476-484.

NAvoN, D. (1977). Forest before trees? The precedence of global features in visual perception. Cognitive Psychology, 9, 353-383.

Navon, D. (1981). The forest revisited: More on global precedence. Psychological Research, 43, 1-32.

NeISSER, U. (1967). Cognitive psychology. New York: AppletonCentury-Crofts.

Pomerantz, J. R. (1981). Perceptual organization in information processing. In M. Kubovy \& J. R. Pomerantz (Eds.), Perceptual organization (pp. 141-179). Hillsdale, NJ: Erlbaum.

Pomerantz, J. R. (1983). Global and local precedence: Selective attention in form and motion perception. Journal of Experimental Psychology: General, 112, 516-540.

Prinzmetal, W. (1981). Principles of feature integration in visual perception. Perception \& Psychophysics, 30, 330-340.

Prinzmetal, W., Millis-Wright, P. (1984). Cognitive and linguistic factors affect visual feature integration. Cognitive Psychology, 16, 305-340.

SChnore, M. M., \& PARTington, J. T. (1967). Immediate memory for visual patterns: Symmetry and amount of information. Psychonomic Science, 8, 421-422.

Sebrechts, M. M., \& Fragala, J. J. (1985). Variations on parts and wholes: Information precedence vs. global precedence. Proceedings of the Seventh Annual Conference of the Cognitive Science Society, 11-18. 
Sebrechts, M. M., \& GARNER, W. R. (1981). Stimulus-specific processing consequences of pattern goodness. Memory \& Cognition, 9, 41-49.

Treisman, A., Gelade, G. (1980). A feature-integration theory of attention. Cognitive Psychology, 12, 97-136.

Treisman, A., Schmidt, H. (1982). Illusory conjunctions in the perception of objects. Cognitive Psychology, 14, 107-141.

Treisman, A., Sykes, M., \& Gelade, G. (1977). Selective attention and stimulus integration. In S. Dornic (Ed.), Attention and performance VI (pp. 333-361). Hillsdale, NJ: Erlbaum.

Uhr, L. (1966). Pattem recognition. New York: Wiley.

WARD, L. M. (1983). On processing dominance: Comment on Pomerantz. Journal of Experimental Psychology: General, 112, 541-546.

WEYNmouth, F. W. (1958). Visual sensory units and the minimal angle of resolution. American Joumal of Ophthalmology, 46, 102-113.

\section{NOTES}

1. It is important to note that spacing and pattern goodness are independent factors. That is, a good global pattern can be dense and the same pattern can also be sparse. Thus, even though density can also influence the speed with which global patterns are processed, it is not considered to reflect pattem goodness. Changing the density of a group of objects does not necessarily change the nature of the group's arrangement, and vice versa. If we consider the ease with which objects come together to form a global pattern as their cohesiveness, then, essentially. pattern goodness can be considered as a reflection of the quality of cohesion, and density can be considered as a reflection of the intensity (or strength) of cohesion. On the other hand, density and distance are intrinsically related: the term density refers to cohesion of a global unit, and the term distance to the amount of space between local units independent of any global structure. Varying the distance of objects in a group varies the density of the group, and vice versa.

2. Correct performance on target-present trials does not necessarily reflect accuracy of correct feature identification and integration. Because of the nature of the target-present stimuli, which involved both a conjunction and a single fearure (in order not to allow subjects to fall back on a possible strategy of detecting the number of filled circles in the display), the rates of correct target detection may also include trials in which illusory conjunctions and feature errors occurred.

(Manuscript received February 14, 1990; revision accepted for publication October 5,1990 .) 